\title{
Experimentally validated FEA models of HF2V damage free steel connections for use in full structural analyses
}

\author{
Jonathan Desombre ${ }^{1}$, Geoffrey W. Rodgers ${ }^{1}$, Gregory A. MacRae ${ }^{2}$, Timon Rabczuk ${ }^{1}$, \\ Rajesh P. Dhakal ${ }^{2}$ and J. Geoffrey Chase*1 \\ ${ }^{1}$ Department of Mechanical Engineering, University of Canterbury, Private Bag 4800, \\ Christchurch 8140, New Zealand \\ ${ }^{2}$ Department of Civil Engineering, University of Canterbury, Private Bag 4800, \\ Christchurch 8140, New Zealand
}

(Received November 20, 2009, Accepted October 21, 2010)

\begin{abstract}
The aim of this research is to model the behaviour of recently developed high force to volume (HF2V) passive energy dissipation devices using a simple finite element (FE) model. Thus, the end result will be suitable for use in a standard FE code to enable computationally fast and efficient analysis and design. Two models are developed. First, a detailed axial model that models an experimental setup is created to validate the approach versus experimental results. Second, a computationally and geometrically simpler equivalent rotational hinge element model is presented. Both models are created in ABAQUS, a standard nonlinear FE code. The elastic, plastic and damping properties of the elements used to model the HF2V devices are based on results from a series of quasi-static force-displacement loops and velocity based tests of these HF2V devices. Comparison of the FE model results with the experimental results from a half scale steel beam-column sub-assembly are within $10 \%$ error. The rotational model matches the output of the more complex and computationally expensive axial element model. The simpler model will allow computationally efficient non-linear analysis of large structures with many degrees of freedom, while the more complex and physically accurate axial model will allow detailed analysis of joint connection architecture. Their high correlation to experimental results helps better guarantee the fidelity of the results of such investigations.
\end{abstract}

Keywords: damage avoidance design; DAD; HF2V; damping; high-force-to-volume; finite element analysis; supplemental damping; experimental; energy dissipation.

\section{Introduction}

Occupant safety and reusability of steel moment-frame buildings following major earthquakes is often limited after major seismic events due to the use of non-linear yielding at structural connections to dissipate energy and response - a life safety focused aspect of typical structural design. By permitting damage to occur in the structural frame system lives are saved but direct structural and economic impacts are enhanced. In particular, damage is primarily restricted to plastic

*Corresponding author, Professor, E-mail: geoff.chase@canterbury.ac.nz 
hinge zones located at the beam ends or the panel zone within steel beam-column joints, which can be difficult, expensive and time consuming to check and repair after a major event. As evidenced by the extensive damage to steel structures following the 1994 Northridge earthquake, repair costs and downtime can be substantial to owners and users of such structures, creating a significant, long-term social and economic impact. It would thus be beneficial to have damage-free connections that still mitigated the same, or greater, amount of energy, removing the need for expensive repairs and enabling business or critical infrastructure, such as hospitals, to operate after earthquakes.

The use of supplementary high force-to-volume (HF2V) devices fitted directly into beam-column connections provides a Damage Avoidance Design (DAD) structural connection (Rodgers et al. 2007). Lead has been used in the first prototypes of these emerging devices due to its unique rheological properties, low re-crystallisation temperature and ability to allow residual compression forces in the damper device to creep back towards zero over time. With these devices, the same, or greater, energy can be dissipated on successive cycles without causing permanent damage. In contrast, conventional steel connections or sacrificial dissipators can only provide such maximum dissipation on 1-2 cycles and much less on subsequent cycles (Bradley et al. 2008, Li 2006), resulting in greater damage and consequently a greater loss than if they were at maximum capacity on all cycles.

Recent advances in these devices have been made by Rodgers et al. (2007, 2008a). HF2V devices

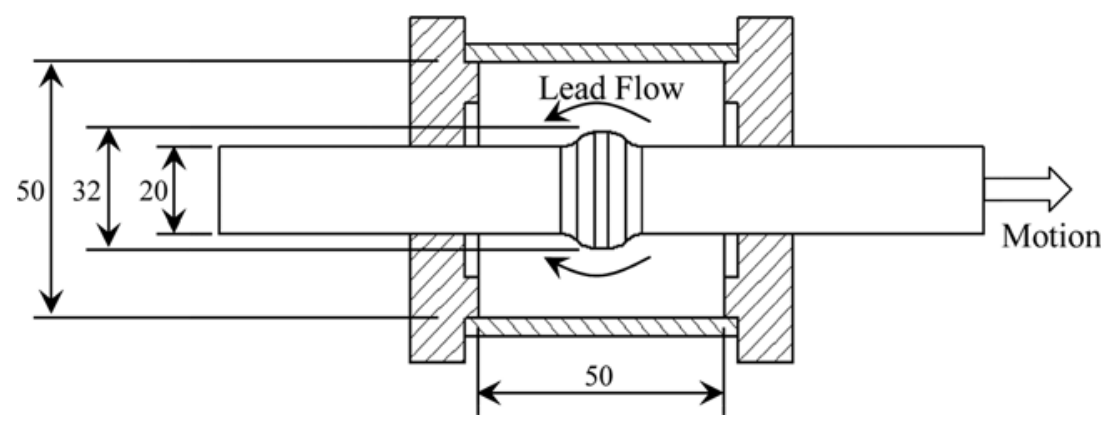

Fig. 1 Cross-sectional view of the HF2V device used in the beam-column joint

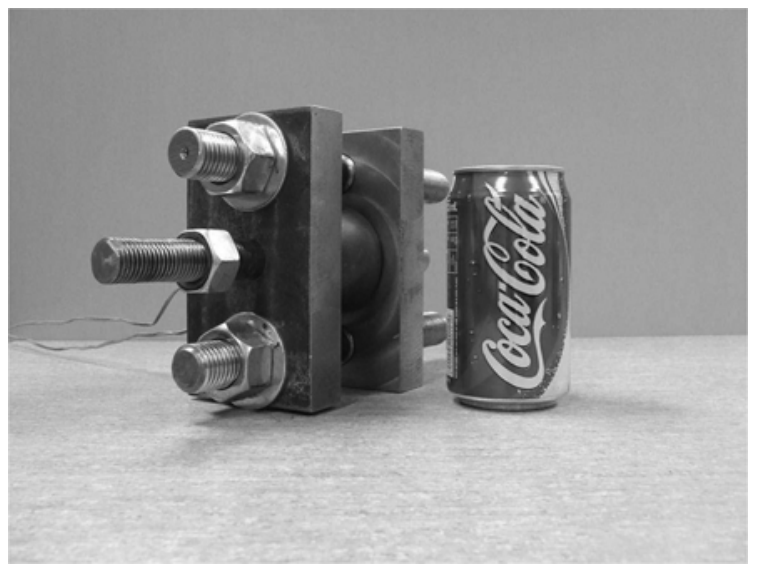

Fig. 2 A $120 \mathrm{kN} \mathrm{HF} 2 \mathrm{~V}$ device with a common item to indicate scale and illustrating its ability to easily fit within a standard structural connection 
using a bulged shaft in a pre-stressed lead cylinder have been developed and experimentally characterized (Rodgers et al. 2007, Rodgers et al. 2008a). Fig. 1 shows a cross-section of a prototype device. These LED devices are inexpensive to manufacture, potentially making them economically feasible for use in a large building. Fig. 2 shows a picture of a $120 \mathrm{kN}$ prototype device.

Similar damping devices have been the subject of previous research including the work of Robinson and Greenbank $(1975,1976)$. These devices were quite large and developed relatively modest forces. They were designed to absorb energy during an earthquake in a controlled manner, with repeatable behaviour. Prototype devices of similar size have been used in several bridges and buildings in New Zealand (Skinner et al. 1993). A summary of this state-of-the-practice that developed from this early work is given by Cousins and Porritt (1993). The major difference with these devices is their compact size, which enables them to be placed directly into typical connections. In addition, spectral analysis has led to the development of performance-based design guidelines to characterize their impact on seismic response for use in design (Rodgers et al. 2008a).

HF2V devices have been implemented in tests, both inside or external, to an $80 \%$ full scale 3D jointed precast concrete connection, where Fig. 3 shows the external test case with devices circled (Rodgers et al. 2008b, Solberg 2007). The devices have also been successfully tested in a 50\% full scale steel beam-column joint, as seen in Fig. 4. This latter experiment used two external devices (north and south side) where only one device is visible in Fig. 4. Fig. 5 shows the hysteretic behaviour of the individual devices and also the behaviour of the overall steel assembly for two fully reversed cycles at 1, 2, 3 and 3.5\% story drift for the steel test case with dampers inside the

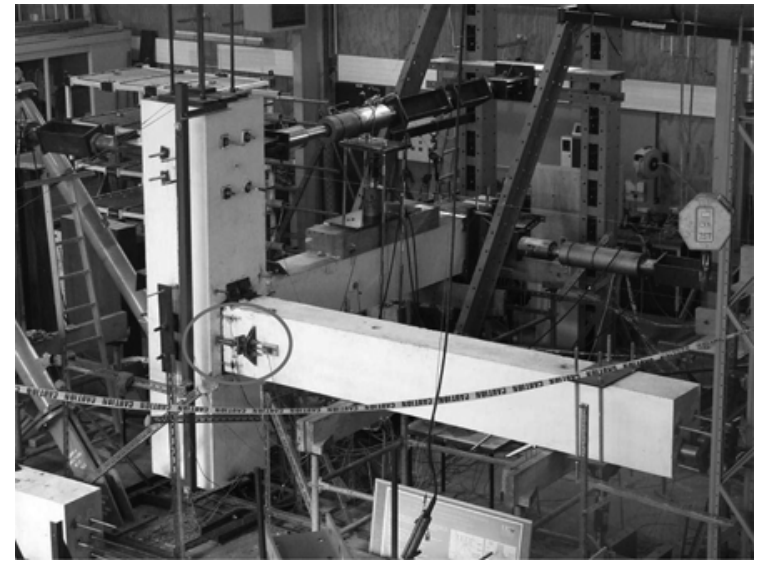

Fig. 3 The jointed precast concrete corner joint experiment utilising HF2V energy dissipation (circled)

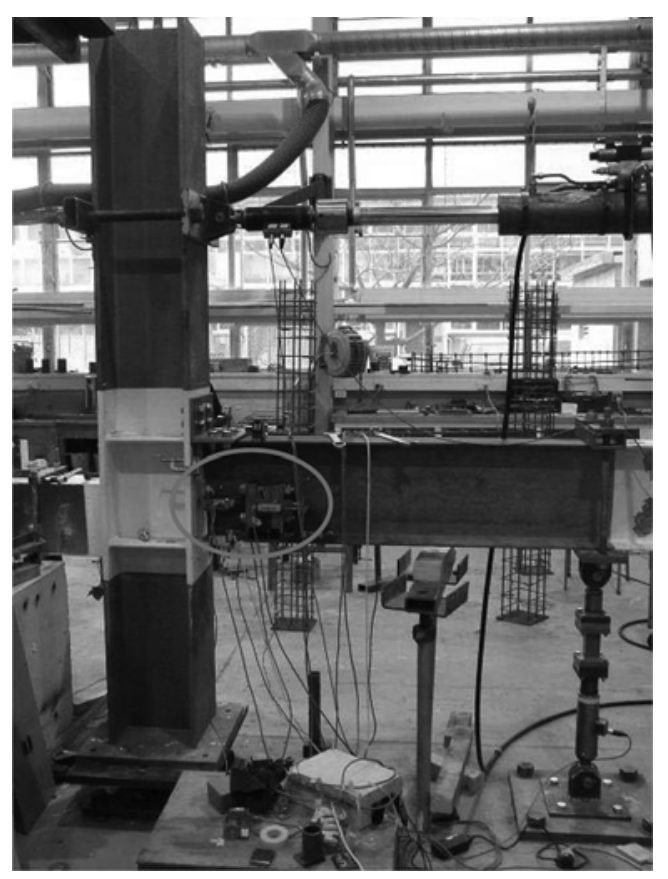

Fig. 4 Steel beam-column experimental setup showing the HF2V devices (circled) 

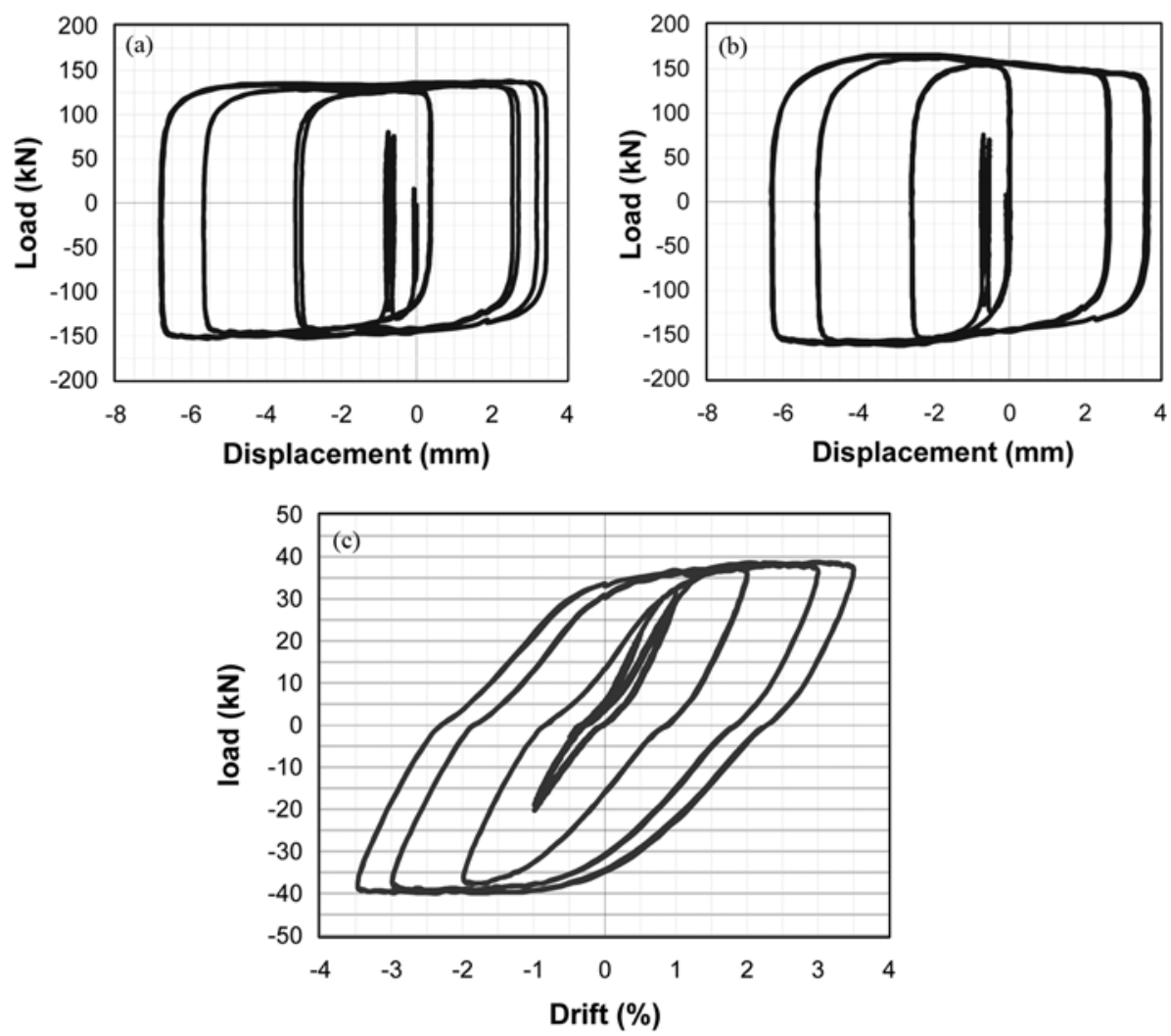

Fig. 5 Hysteresis loops of (a) the North damper, (b) the South damper, and (c) the overall joint subassembly

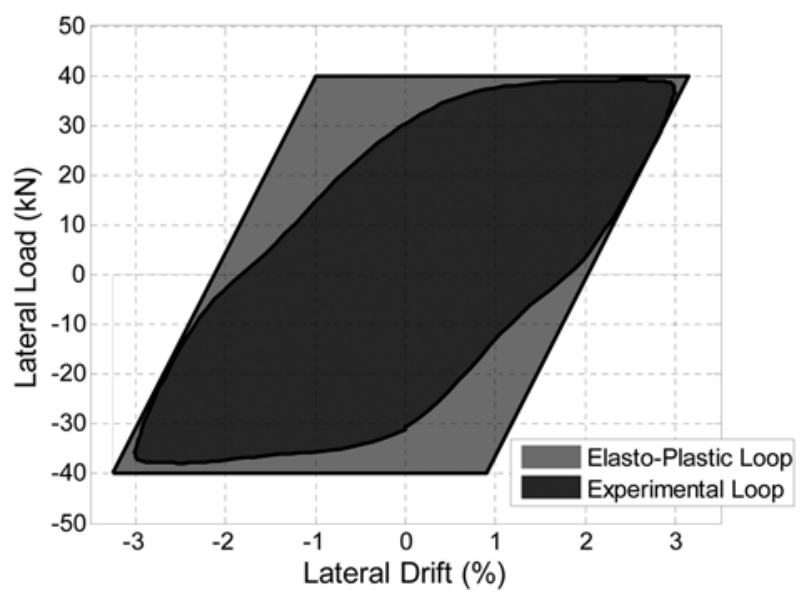

Fig. 6 Energy dissipation efficiency compared to an elastic-perfectly plastic hysteresis loop

beam cross-section (Fig. 4).

Energy dissipation efficiency maybe computed as the ratio of the energy dissipated in one cycle of the subassembly to that of an elastic perfectly plastic loop with the same initial stiffness as the 
device. As shown in Fig. 6, the devices provide an $80 \%$ efficient dissipation at $3 \%$ drift. In contrast, at $3 \%$ drift a standard rigid steel connection will suffer permanent damage, and is only $60 \%$ efficient on that cycle, with lower efficiency on subsequent cycles. The DAD steel joint was completely undamaged at $4 \%$ drift. Similar results are obtained for the concrete connections, compared to similar reinforced concrete connections, for the tests in Fig. 3 (Rodgers et al. 2008b, Solberg 2007). Hence, using these devices can provide more efficient energy dissipation without yielding damage to the connection, and can do so every cycle regardless of prior motions or drifts. More succinctly, they can provide greater dissipation without damage than standard connections.

This research develops non-linear analytical models for the devices and the steel beam-column joint of Fig. 4. The purpose of the research is to produce a numerical model for a standard finite element analysis (FEA) programme that accurately captures the non-linear device and connection behaviour. This device modelling approach is needed to better investigate the application of these devices in full scale building design, in terms of architecture and layout. Thus, a simple model would enable computationally efficient design analysis using standard codes, a significant contribution to seeing the application of these, and similar, devices.

Many different types of dampers and devices have been considered for both retrofit and seismic control of moment frame structures, and are well reviewed (Chen and Ding 2008, Martinez-Rodrigo and Romero 2003, Parulekar and Reddy 2009). Specific studies of non-linear viscous dampers, like the HF2V devices utilised here, and similar visco-elastic devices, include (Amadio et al. 2008, Lin and Chopra 2003a, b, c, Li and Liang 2007, Silvestri et al. 2003). In particular, these HF2V devices are velocity and displacement dependent similar to works studying these effects and similar devices by (Chang et al. 2001, Pong et al. 2002). In addition, the overall concept of reducing steel connection stiffness to induce motion into a separate energy dissipation device has also been previously studied conceptually (Reyes-Salazar and Haldar 2000). Finally, given the experimental proof of concept and the finite element models presented above, designers and research studies may more closely examine the optimal placement of these devices within a given structure, which is a well studied area in its own right (Desu et al. 2007, Marano et al. 2007). However, it is worth noting that no studies have sought to capture the critical non-linear dynamics of these devices in models suitable for use in large finite element models to design full structural systems.

Hence, the FE model created in this work should be as simple as possible to enable its use in a large structure model. A typical example of the structure that might be analysed or designed with these devices would include the well known SAC3 and SAC9 frames designed for Los Angeles (also known as LA3 and LA9). Simplicity will minimize the computational demand of non-linear FEA time-history analysis over several ground motions or suites of ground motions per emerging performance based design approaches. The model presented here will thus enable effective design in conjunction with the previously developed spectral analysis results (Rodgers et al. 2008a).

\section{Methods}

The goal of this research is to create a simple numerical model of a steel beam-column joint with, in this case, two HF2V devices. Thus, a finite element model of a steel beam-column connection with HF2V devices is developed and verified based on experimental result from a half-scale subassembly test. 


\subsection{Steel joint experimental setup}

The experimental setup is shown in Fig. 7. Repeated experiments were performed from $0.25 \%$ to $4.0 \%$ drift. All results and hysteretic loops were consistently repeatable, as seen in Figs. 5, 6. The displacement of the top of the column is controlled by the hydraulic actuator to apply a fully reversed sinusoidal displacement (i.e., drift) history, a sample of which is illustrated in Fig. 8. Hence, the drifts are both increased and then decreased to show energy dissipation on subsequent smaller drift cycles. Sensors record forces and displacements in the joint. Fig. 5(c) shows results for typical reversed cyclic load sequences up to $3.5 \%$ drift. The hysteretic loops of the north and south devices are also shown in Figs. 5(a),(b).

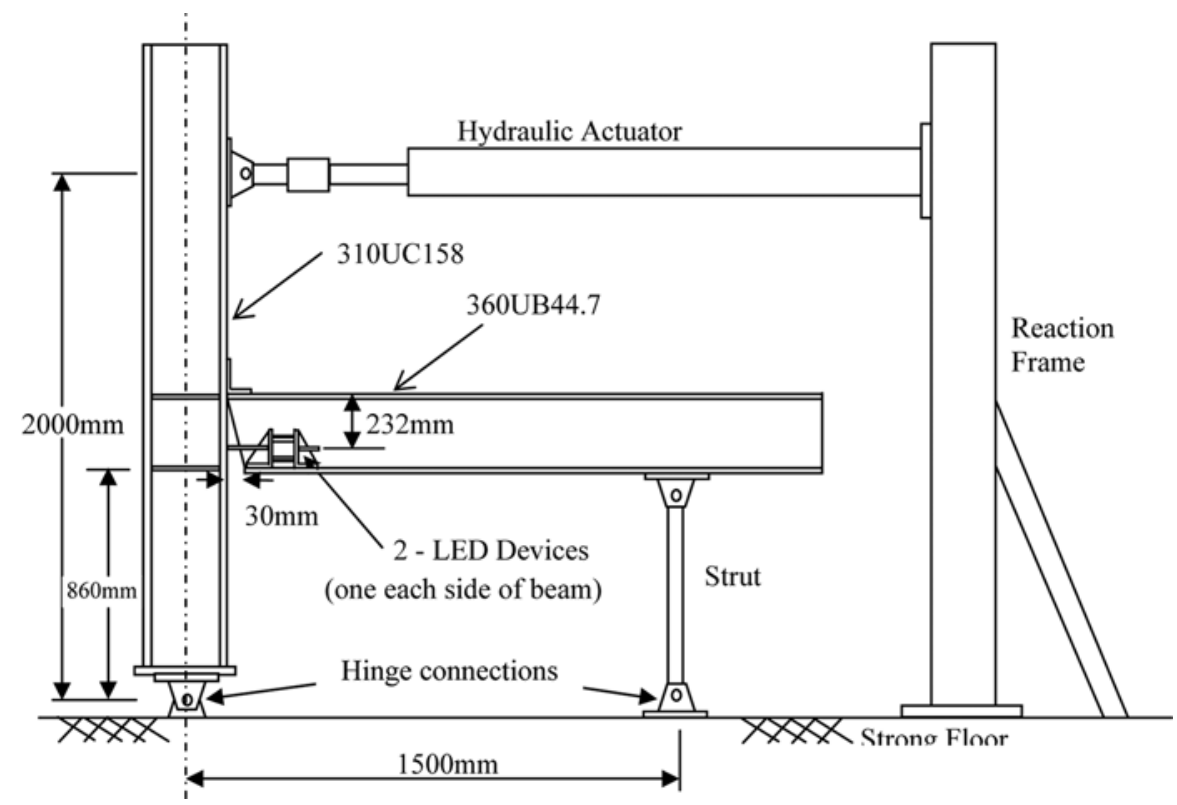

Fig. 7 Experimental set-up of the exterior steel beam-column joint with the non-dilating rigid connection designed for damage avoidance, as viewed looking North from the (visible) South side of the experimental setup

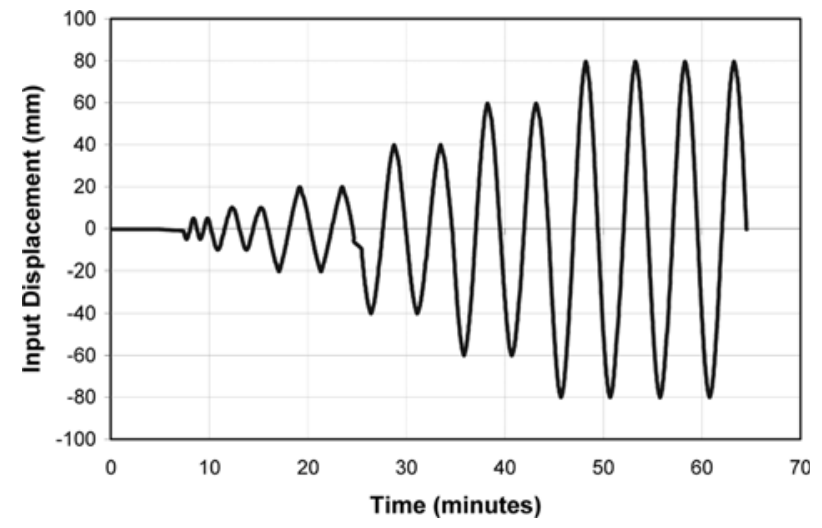

Fig. 8 Experimental time-displacement input data, where $80 \mathrm{~mm}$ displacement corresponds to $4 \%$ drift 


\subsection{Finite element model}

A 2D analysis in ABAQUS is used to model the sub-assembly experiment. The beam and columns are modelled with beam linear elements (B31) with a profile definition according to the universal column 310 UC 158 and universal beam 360 UB 44.7 measurements. The strut is modelled with rigid elements. The pins connections have been modelled with perfect hinge connector elements. The time-displacement history applied to the top the column used in the analytical simulation is exactly the same as that used in the experiment, which is shown in Fig. 8.

First, non-linear AXIAL connector elements are used to model the devices to correlate the experiment and numerical model. Once this model matches the experimental result, the AXIAL connectors and the inclined rigid element are converted into a rotational HINGE model to make the numerical analysis much simpler to use in large FEA simulations.

\subsection{AXIAL model}

The first models of the beam-column connection, including the devices, use a rigid element connected to the column with a perfect hinge at one end. The other end of the rigid element is connected to the devices, which are represented by a non-linear spring and a non-linear dashpot in parallel as shown in Fig. 9(b). The beam is connected to the rigid element with a rigid connection at the "neutral fibre" position of the beam. Figs. 9(a),(b) show the model and device model layouts in schematic form. As shown in Fig. 9(d), the length and inclination of the inclined rigid element is determined from the experimental subassembly. The rigid line connects the column centreline at the hinge point, to the centre of the HF2V damper.

The devices are modelled with AXIAL connector element with the nonlinear mechanical properties of the HF2V devices. The elasticity, plasticity and damping properties of the device, as determined experimentally, are implemented in the axial connector element definition. These experimental device results are obtained from the experimental data contained in the square hysteretic loops shown in Figs. 5(a),(b).

This first model, Model 1, does not account for the elasticity of the device shaft. Moreover, almost a third of the beam length is occupied by the rigid element used to model the joint. The second model, Model 2 shown in Fig. 9(c), has a corrective spring added in series with the device. The stiffness of these springs is chosen to match the shaft elasticity measured in the experimental results. The added spring is then merged in the AXIAL connector properties by reducing the elastic stiffness of the nonlinear spring in the device of Fig. 9(b), rather than having individual springs in series with the devices.

\subsection{HINGE model}

By modelling the joint with an equivalent rotational HINGE connector element, the number of degrees of freedom can be reduced sufficiently to be more easily used in large building analyses with minimal added degrees of freedom or computational cost. The HINGE connector element is acts like a non-linear rotational spring with non-linear damping to mimic the axial device properties based on the moment arm of its connection in the experimental setup of Fig. 7. The resulting equivalent ABAQUS model is shown in Fig. 10. 


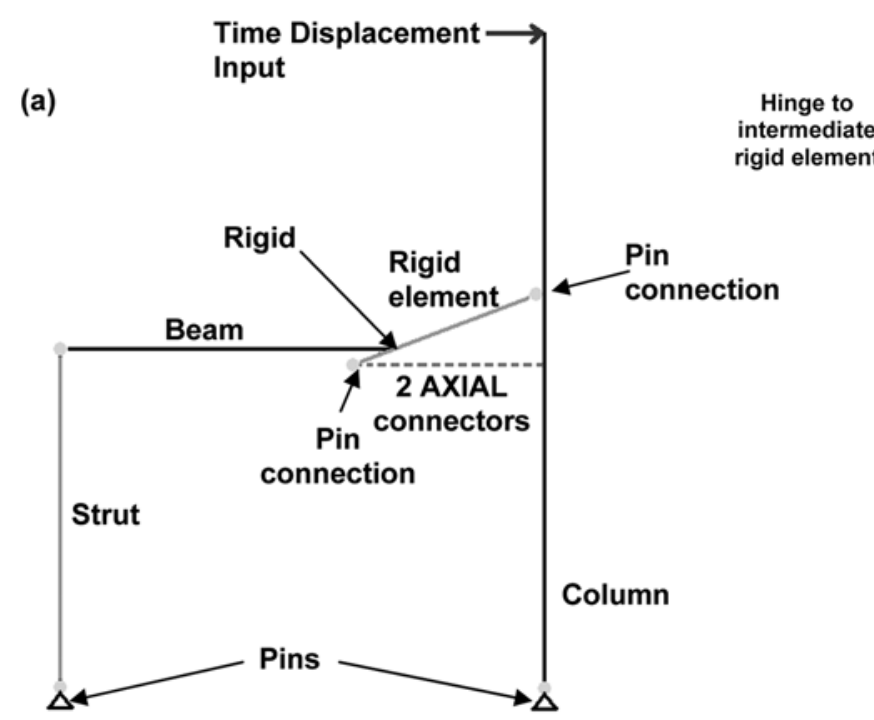

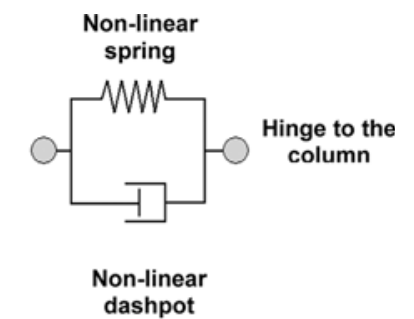

(b) Model 1

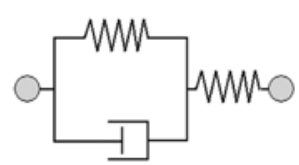

(c) Model 2

(d) Model parameters

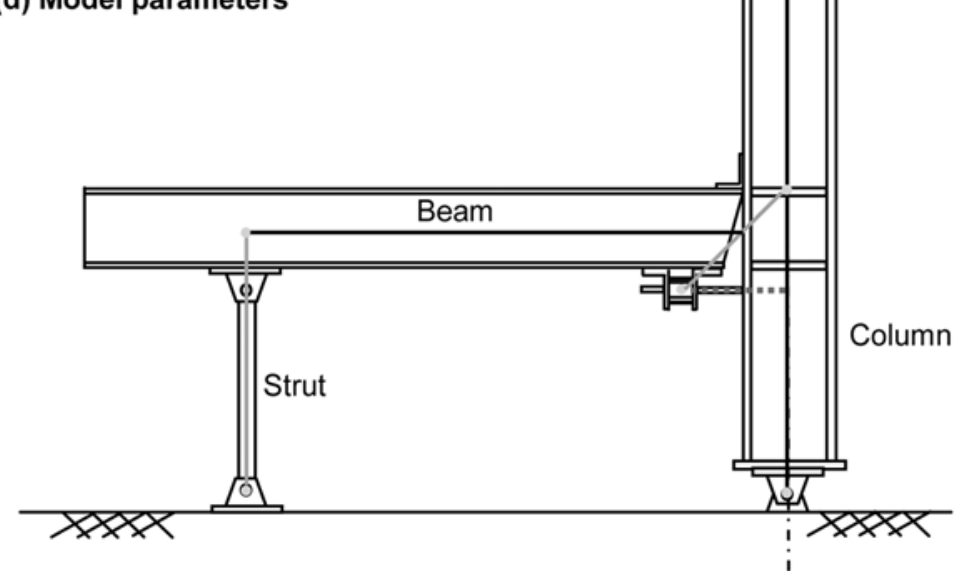

Fig. 9 ABAQUS AXIAL model configuration (a) (not to scale) and axial device models (b,c) which occupy the elements in the dashed line in panel (a) labelled "AXIAL connectors", (d) shows the schematic overlay of the model and experimental joint

\subsection{Specific device parameters}

The device parameters used in the FEA models for this experiment come from two main series of tests. A series of quasi-static force-displacement hysteretic loops of each device and a series of velocity based tests for each device to quantify for their velocity dependence (Rodgers et al. 2008a). 


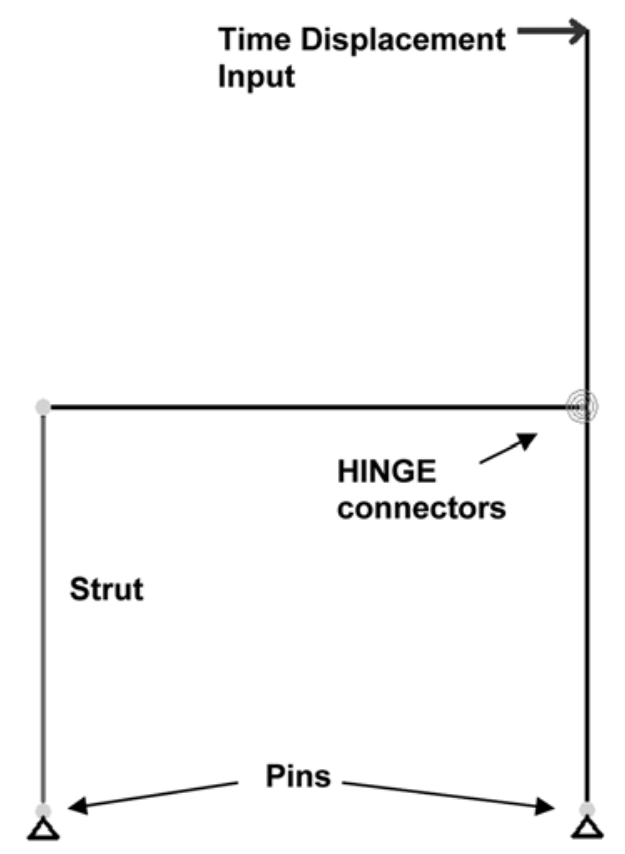

Fig. 10 ABAQUS HINGE model

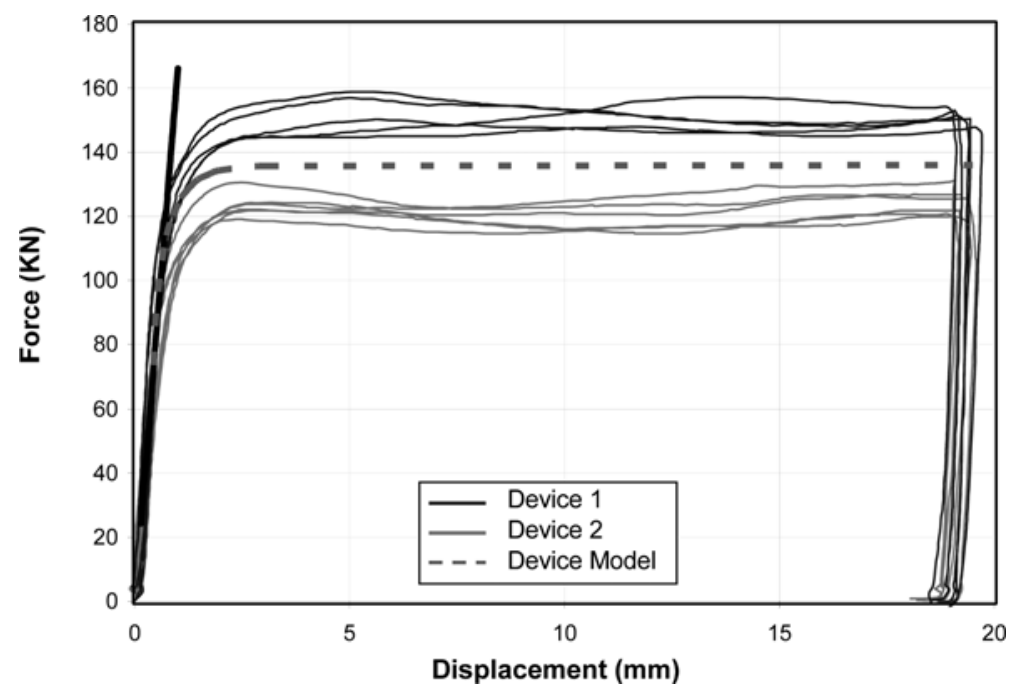

Fig. 11 Response of the two devices and the fitted model for use in ABAQUS

Fig. 11 shows a portion of the quasi-static test results for both devices. They don't have strictly the exact same level of maximum force, but they have the same force-displacement curve shape. An average force-displacement curve is used in the model as shown by the dashed line in Fig. 11. The elastic behaviour is the same for both devices, as seen in the almost vertical solid line. This average device stiffness, which is used in the model, is $k_{\text {device }}=165 \times 10^{6} \mathrm{~N} / \mathrm{m}$. 
Fig. 12 show the hysteretic loops and input velocity profile for testing different velocities. Fig. 13 plots peak force at zero displacement on the $x$-axis versus velocity on the y-axis from the data in Fig. 12. Note that Fig. 13 has the quasi-static force at approximately zero velocity subtracted so that only contribution to force from added velocity is considered. This subtraction removes the static base device force $\left(D_{o}\right)$ from a series of tests on different devices with different $D_{o}$ values (Rodgers et al. 2007). The resulting data and curves represent the added force due to velocity, above the static yielding force $D_{o}$.

A line is fitted to the Fig. 13 experimental results and used to add the velocity dependant damping contribution in the connector (AXIAL/HINGE) elements that this effect is captured by the FEA modelled device. Thus, the FEA device model now accounts for the shaft compliance and the velocity dependent force; $F_{d}=C_{0} \dot{x}^{\alpha}$ where $\alpha=0.11$ (Rodgers et al. 2008a). Hence, the
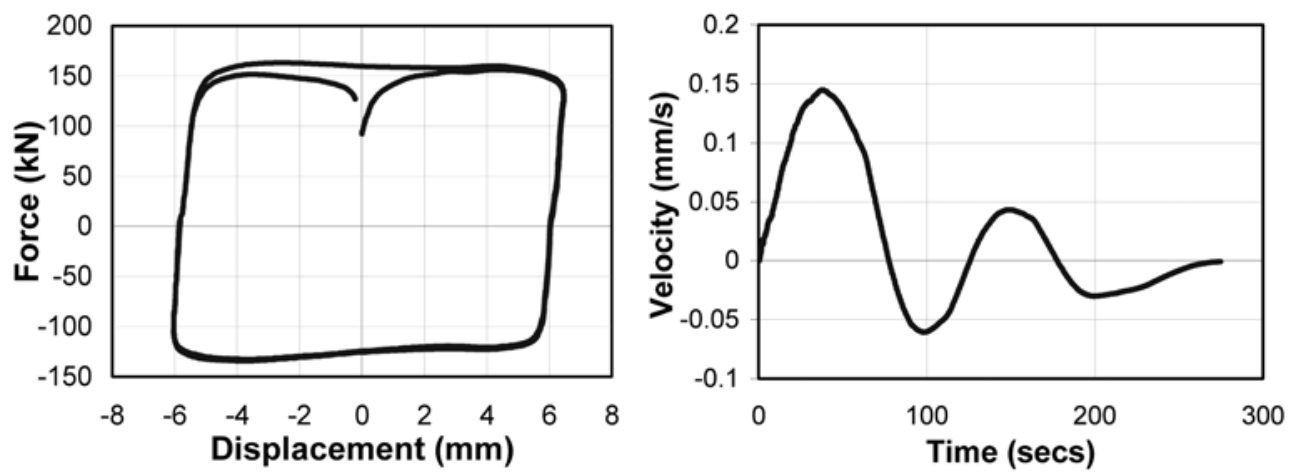

Fig. 12 Force-displacement results of HF2V dampers during velocity tests (left) and velocity profile (right)

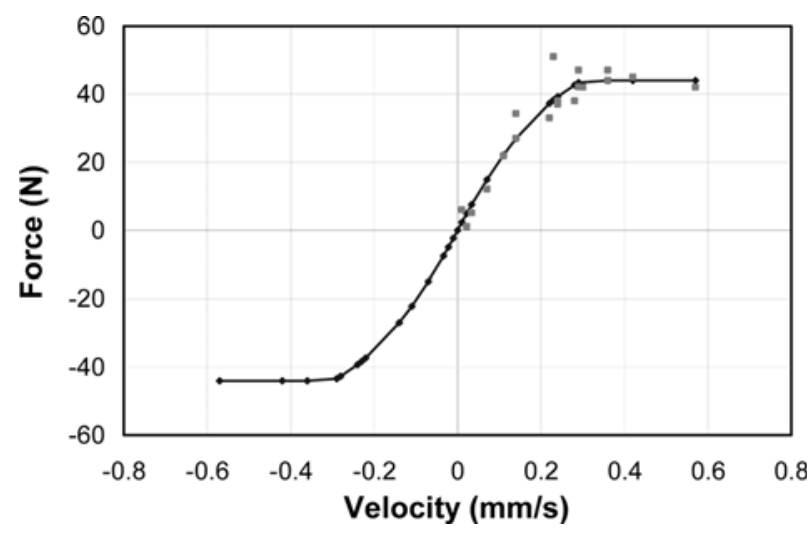

Fig. 13 The damping force $\left(F_{v e l}\right)$ contribution to total device force

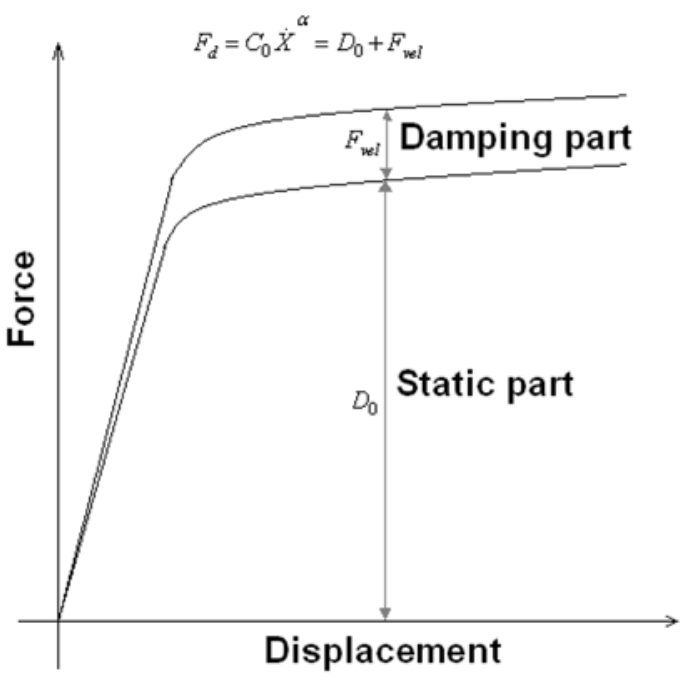

Fig. 14 Device model force-displacement relationship resulting from static and velocity dependent contributions 
total device force is modelled in ABAQUS in two portions: $F_{d}=C_{0} \dot{x}^{\alpha}=D_{0}+F_{v e l}$ as shown in Fig. 14.

\subsection{Model parameter summary}

In ABAQUS the devices are modelled with connector elements. The behaviour of these elements is defined by several parameters. In these models, the behaviours of the connector element are defined with 3 parameters:

1. Elasticity $\rightarrow$ Linear and uncoupled.

2. Plasticity $\rightarrow$ Non-linear and uncoupled, using a kinematic hardening model with a half-cycled definition (ABAQUS manual, Lee et al. 2008).

3. Damping $\rightarrow$ Non-linear and uncoupled.

The specific values that resulted in a good match to the experimental data are shown in Table 1, which is provided in an Appendix for clarity.

Table 1 Input parameters for ABAQUS models

\begin{tabular}{|c|c|c|c|}
\hline \multicolumn{2}{|c|}{$\begin{array}{c}\text { Connector element type: AXIAL } \\
\text { Plasticity }\end{array}$} & \multicolumn{2}{|c|}{$\begin{array}{c}\text { Connector element type : HINGE } \\
\text { Plasticity }\end{array}$} \\
\hline Plasticity & Nonlinear & Plasticity & Nonlinear \\
\hline Specify Kinematic & Uncoupled & Specify Kinematic & Uncoupled \\
\hline \multicolumn{2}{|c|}{ Definition : Half - cycle } & \multicolumn{2}{|c|}{ Definition : Half - cycle } \\
\hline $\mathrm{F}[\mathrm{N}]$ & Plastic Motion $[\mathrm{mm}]$ & Angle $\left(\operatorname{rad} \times 10^{-3}\right)$ & Moment (N.m) \\
\hline 60240 & 0 & 0.000 & 13976 \\
\hline 72170 & 0.044 & 0.190 & 16743 \\
\hline 82960 & 0.112 & 0.483 & 19247 \\
\hline 92700 & 0.202 & 0.871 & 21506 \\
\hline 100500 & 0.306 & 1.319 & 23316 \\
\hline 106700 & 0.456 & 1.966 & 24754 \\
\hline 112100 & 0.635 & 2.737 & 26007 \\
\hline 115000 & 0.807 & 3.478 & 26680 \\
\hline 118000 & 1.039 & 4.478 & 27376 \\
\hline 119500 & 1.279 & 5.513 & 27724 \\
\hline 120800 & 1.518 & 6.543 & 28026 \\
\hline 122100 & 2.356 & 10.155 & 28327 \\
\hline \multirow[t]{2}{*}{122200} & 2.61 & 11.250 & 28350 \\
\hline & & $\arctan (m o t i o n / 0.232)$ & force $^{*} 0.232$ \\
\hline
\end{tabular}


Table 1 Continued

\begin{tabular}{|c|c|c|c|}
\hline \multicolumn{2}{|c|}{ Elasticity } & \multicolumn{2}{|c|}{ Elasticity } \\
\hline $\mathrm{K}=165 \times 10^{6} \mathrm{~N} / \mathrm{m}$ & Device alone & $\mathrm{K}=3.77 \times 10^{6} \mathrm{~N} . \mathrm{m} / \mathrm{rad}$ & Un-corrected \\
\hline $\mathrm{K}=80 \times 10^{6} \mathrm{~N} / \mathrm{m}$ & in Sub-assembly & $\mathrm{K}=4.5 \times 10^{6} \mathrm{~N} \cdot \mathrm{m} / \mathrm{rad}$ & Corrected \\
\hline \multicolumn{2}{|c|}{ Damping } & \multicolumn{2}{|c|}{ Damping } \\
\hline Nonlinear & Uncoupled & Nonlinear & Uncoupled \\
\hline Force $(\mathrm{N})$ & Velocity $(\mathrm{mm} / \mathrm{s})$ & Moment (N.m) & Velocity $\left(\mathrm{rad} / \mathrm{s} \times 10^{-3}\right)$ \\
\hline-44070 & -0.570 & -10224 & -2.457 \\
\hline-44070 & -0.420 & -10224 & -1.810 \\
\hline-44070 & -0.360 & -10224 & -1.552 \\
\hline-43424 & -0.290 & -10074 & -1.250 \\
\hline-42721 & -0.280 & -9911 & -1.207 \\
\hline-39342 & -0.240 & -9127 & -1.034 \\
\hline-38356 & -0.230 & -8899 & -0.991 \\
\hline-37313 & -0.220 & -8657 & -0.948 \\
\hline-26923 & -0.140 & -6246 & -0.603 \\
\hline-22090 & -0.110 & -5125 & -0.474 \\
\hline-14852 & -0.070 & -3446 & -0.302 \\
\hline-7561 & -0.034 & -1754 & -0.147 \\
\hline-4967 & -0.022 & -1152 & -0.095 \\
\hline-2292 & -0.010 & -532 & -0.043 \\
\hline 0 & 0.000 & 0 & 0.000 \\
\hline 2292 & 0.010 & 532 & 0.043 \\
\hline 4967 & 0.022 & 1152 & 0.095 \\
\hline 7561 & 0.034 & 1754 & 0.147 \\
\hline 14852 & 0.070 & 3446 & 0.302 \\
\hline 22090 & 0.110 & 5125 & 0.474 \\
\hline 26923 & 0.140 & 6246 & 0.603 \\
\hline 37313 & 0.220 & 8657 & 0.948 \\
\hline 38356 & 0.230 & 8899 & 0.991 \\
\hline 39342 & 0.240 & 9127 & 1.034 \\
\hline 42721 & 0.280 & 9911 & 1.207 \\
\hline 43424 & 0.290 & 10074 & 1.250 \\
\hline 44070 & 0.360 & 10224 & 1.552 \\
\hline 44070 & 0.420 & 10224 & 1.810 \\
\hline \multirow[t]{2}{*}{44070} & 0.570 & 10224 & 2.457 \\
\hline & & force $^{*} 0.232$ & $\arctan ($ Velocity/0.232) \\
\hline
\end{tabular}




\section{Results and discussion}

Fig. 15 shows experimental results (solid) and the ABAQUS Model 1 results (dashed). The $x$-axis is the drift in percentage of the top of the column, where the actuator is attached. The $y$-axis is the reaction force in $\mathrm{kN}$ at the same position. Thus, Fig. 15 is an overall beam column joint hysteretic loop. There is an obvious and significant difference in the unloading and reloading stiffness properties, however the maximum force is close the experimental value. The difference in stiffness can be explained by the fact that Model 1 does not capture the compliance of the device shaft. In addition, the rigid element employed covers almost $30 \%$ of the beam, which makes the numerical model stiffer than the experiment.

The corrected AXIAL model (Model 2) is shown in Fig. 16. The results now match the elastic behaviour of the experimental results much more closely due to the net correction for the rigid element and axial device stiffness.

Fig. 17 compares the corrected AXIAL model (Model 2) and the equivalent rotational HINGE model (Model 3), showing very close matches.

\subsection{Main differences}

The experimental results in Figs. 15-17 show that each time the experimental curve crosses the $x$ -

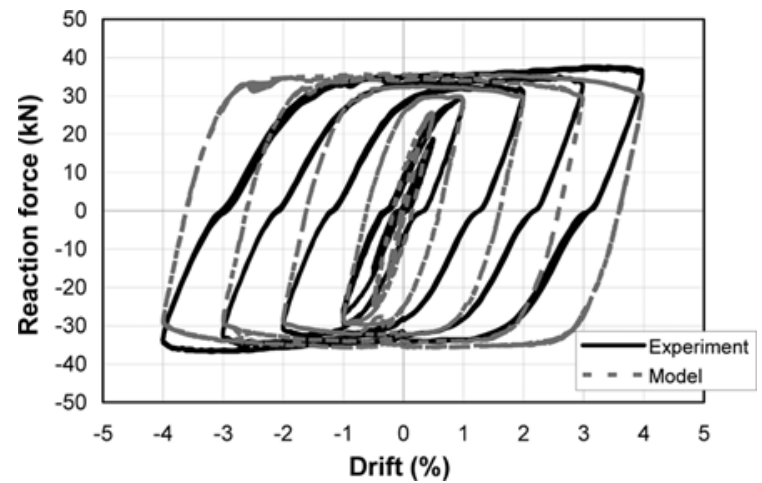

Fig. 15 Experimental and Model 1 results overlaid

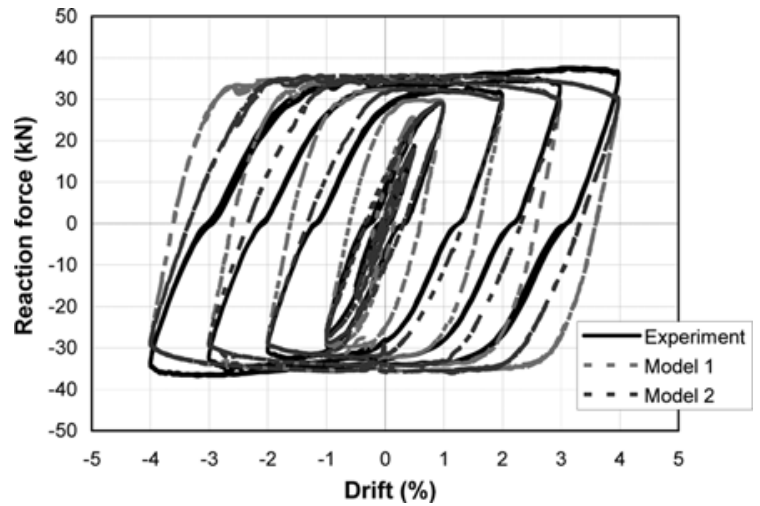

Fig. 16 Experimental results versus Models 1 and 2 overlaid

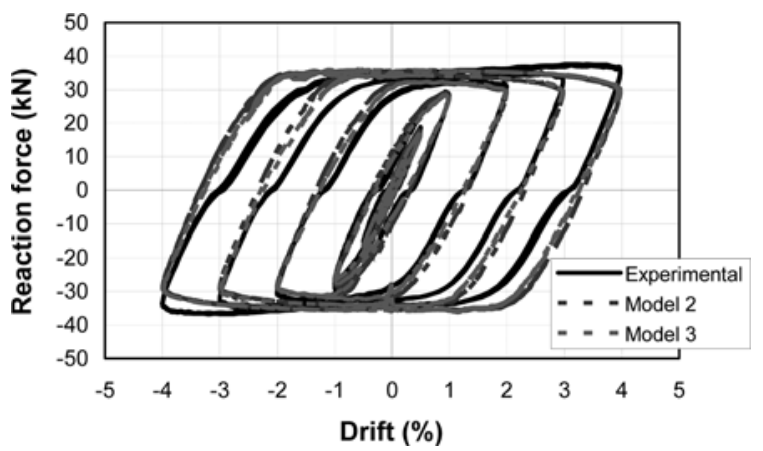

Fig. 17 Experimental results versus Models 2 and 3 overlaid 
axis there is a small amount of slip. This slip is due compliance in the sub-assembly bracket connections that creates a small slip in the data when motion is reversed. Note that Figs. 5(a),(b) show no such slip in the experiments on the devices in service, indicating the that this difference must be due to compliance in the connections to the beams. This phenomenon was not modelled because the effect is small and primarily due to an under designed experimental bracket.

The ABAQUS results also show that the reaction force decreases just before unloading. This drop off from the FEA results is explained by the fact that the imposed displacements are not perfectly triangular, but are smooth at the peaks. Hence, the velocity is decreasing to zero before unloading starts. Therefore, the damping force contribution is decreasing, and thus the global reaction force decreases. Again this difference is one of the specific experimental procedure and implementation, rather than realistic in-situ behaviour, and was thus ignored.

\section{Conclusions}

This research has produced two experimentally validated finite element models to represents the behaviour of non-linear HF2V devices in steel connections. They are a first step in enabling the modelling and analysis of full scale non-linear structural response, to prove the concept and potential advantages of these, and similar, devices before larger trials or tests. A non-linear rotational spring with non-linear damping model (Model 3) enables use in large degree-of-freedom non-linear structural models due to its lower computational complexity and reduced number of added degrees of freedom compared to the more directly modelled axial model (Models 1-2). The development and verification have been made using experimental results from a half-scale steel beam-column join sub-assembly. However, the overall results and approach are generalisable to any structural connection system. In particular, the mechanical properties reported are readily scaled or obtained experimentally to generalise these results to larger, or smaller, similar devices. Hence, the overall results can be readily generalised to broader classes of devices, enabling more straightforward FEA modelling in larger structural systems for ease in overall structural design. Further ongoing research will evaluate the computational cost and complexity, as well as any advantages, of using these models in suites of simulations for full scale structural design and analysis.

\section{References}

Amadio, C., Clemente, I., Macorini, L. and Fragiacomo, M. (2008), "Seismic behaviour of hybrid systems made of PR composite frames coupled with dissipative bracings", Earthq. Eng. Struct. Dyn., 37, 861-879.

Bradley, B.A., Dhakal, R.P., Mander, J.B. and Li, L. (2008), "Experimental multi-level seismic performance assessment of 3D RC frame designed for damage avoidance", Earthq. Eng. Struct. Dyn., 37, 1-20.

Chang, K.C., Tsai, M.H. and Lai, M.L. (2001), "Shaking table study of a 2/5 scale steel frame with new viscoelastic dampers", Struct. Eng. Mech., 11, 273-286.

Chen, Y.H. and Ding, Y.J. (2008), "Passive, semi-active, and active tuned-liquid-column dampers", Struct. Eng. Mech., 30, 1-20.

Cousins, W.J. and Porritt, T.E. (1993), "Improvements to lead-extrusion damper technology", Bul. N.Z. Nat. Soc. Earthq. Eng., 26, 342-348.

Desu, N.B., Dutta, A. and Debt, S.K. (2007), "Optimal assessment and location of tuned mass dampers for 
seismic response control of a plan-asymmetrical building", Struct. Eng. Mech., 26, 459-477.

Lee, H.K., Ha, S.K. and Afzal, M. (2008), "Finite element analysis of shear-deficient RC beams strengthened with CFRP strips/sheets", Struct. Eng. Mech., 30, 247-261.

Li, B. and Liang, X.W. (2007), "Design of supplemental viscous dampers in inelastic SDOF system based on improved capacity spectrum method", Struct. Eng. Mech., 27, 541-554.

Li, L. (2006), "Further experiments on damage avoidance design of beam-to-column joints", Dept. of Civil Engineering, University of Canterbury, Christchurch, New Zealand.

Lin, W.H. and Chopra, A.K. (2003a), "Asymmetric one-storey elastic systems with non-linear viscous and viscoelastic dampers: Earthquake response", Earthq. Eng. Struct. Dyn., 32, 555-577.

Lin, W.H. and Chopra, A.K. (2003b), "Asymmetric one-storey elastic systems with non-linear viscous and viscoelastic dampers: Simplified analysis and supplemental damping system design", Earthq. Eng. Struct. Dyn., 32, 579-596.

Lin, W.H. and Chopra, A.K. (2003c), "Earthquake response of elastic single-degree-of-freedom systems with nonlinear viscoelastic dampers", J. Eng. Mech.-ASCE, 129, 597-606.

Marano, G.C., Trentadue, F. and Greco, R. (2007), "Stochastic optimum design criterion of added viscous dampers for buildings seismic protection", Struct. Eng. Mech., 25, 21-37.

Martinez-Rodrigo, M. and Romero, M.L. (2003), "An optimum retrofit strategy for moment resisting frames with nonlinear viscous dampers for seismic applications", Eng. Struct., 25, 913-925.

Parulekar, Y.M. and Reddy, G.R. (2009), "Passive response control systems for seismic response reduction: a state-of-the-art review", Int. J. Struct. Stab. Dyn., 9, 151-177.

Pong, W.S., Tsai, C.S., Chen, C.S. and Chen, K.C. (2002), "Parametric study for buildings with combined displacement-dependent and velocity-dependent energy dissipation devices", Struct. Eng. Mech., 14, 85-98.

Reyes-Salazar, A. and Haldar, A. (2000), "Dissipation of energy in steel frames with PR connections", Struct. Eng. Mech., 9, 241-256.

Robinson, W.H. and Greenbank, L.R. (1975), "Properties of an extrusion energy absorber", Bul. N.Z. Nat. Soc. Earthq. Eng., 8, 187-191.

Robinson, W.H. and Greenbank, L.R. (1976), "Extrusion energy absorber suitable for the protection of structures during an earthquake", Earthq. Eng. Struct. Dyn., 4, 251-259.

Rodgers, G.W., Chase, J.G., Mander, J.B., Leach, N.C. and Denmead, C.S. (2007), "Experimental development, tradeoff analysis and design implementation of high force-to-volume damping technology", Bul. N.Z. Nat. Soc. Earthq. Eng., 40, 35-48.

Rodgers, G.W., Mander, J.B., Chase, J.G., Dhakal, R.P., Leach, N.C. and Denmead, C.S. (2008a), "Spectral analysis and design approach for high force-to-volume extrusion damper-based structural energy dissipation", Earthq. Eng. Struct. Dyn., 37, 207-223.

Rodgers, G.W., Solberg, K.M., Mander, J.B., Chase, J.G., Bradley, B.A., Dhakal, R.P. and Li, L. (2008b), "Performance of a damage-protected beam-column subassembly utilizing external HF2V energy dissipation devices", Earthq. Eng. Struct. Dyn., 37, 1549-1564.

Silvestri, S., Trombetti, T. and Ceccoli, C. (2003), "Inserting the mass proportional damping (MPD) system in a concrete shear-type structure", Struct. Eng. Mech., 16, 177-193.

Skinner, R.I., Robinson, W.H. and Mcverry, G.H. (1993), An Introduction to Seismic Isolation, Chichester, Wiley, New York.

Solberg, K.M. (2007), "Experimental and financial investigations into the further development of damage avoidance design", Department of Civil Engineering, Christchurch, New Zealand, University of Canterbury. 